\title{
Passive Micro-resonator based GMPLS Router for Optical Network-on-Chip
}

\author{
Suvarna S. Patil \\ Bharati Vidyapeeth University, Pune - 411043, India, \\ Phone no.: +91 9881717562, Email: sschorage@gmail.com
}

Bharat S. Chaudhari

International Institute of Information Technology, Pune - 411057, India

Email id: bharatc@isquareit.ac.in

Received: June 6, $2011 \quad$ Accepted: July 24, $2011 \quad$ DOI: 10.5296/ npa.v3i2.704

\begin{abstract}
Generalized multiprotocol label switching (GMPLS) with wavelength division multiplexing (WDM) technology is a very promising protocol technology for future optical networks. GMPLS technology interconnects new and legacy networks by automating connection provisioning and traffic engineering. The present electrical interconnects for the system on chip $(\mathrm{SoC})$ are unable to satisfy the multiple design requirements of bandwidth, data rate and latency. In this paper, for the first time we have proposed a micro resonator based GMPLS router for optical network on chip $(\mathrm{ONoC})$. The evolution of such a router node on integrated circuit technology will cause the system design to move towards a communication-based architecture. The concept of an integrated GMPLS optical interconnect will be a potential technological solution, alleviating some of the more pressing issues involved in exchanging data between cores in SoC architectures. The investigation for $\mathrm{ONoC}$ was carried out at the physical level, where the system performance on the basis of crosstalk, blocking probability, offered traffic load and packet error rate (PER) was analyzed.
\end{abstract}

Keywords: Blocking probability, crosstalk, GMPLS, ONoC, PER, SoC, WDM. 


\section{Introduction}

Future data transmission networks will consist of elements such as; routers, switches, Dense Wavelength Division Multiplexing (DWDM) systems, Add-Drop Multiplexers (ADMs), photonic cross-connects (PXCs), optical cross-connects (OXCs) etc., that will use Generalized Multi-Protocol Label Switching (GMPLS) to dynamically provision resources and to provide network survivability using protection and restoration techniques. Integrated optical interconnects have a greater potential to solve the issues related to huge data transfers between system components. It enables the high bandwidth and low contention routing of data, using wavelength division multiplexing (WDM) technology. Metallic interconnects are highly inefficient in a high-speed data transfer environment, due to their major limitations such as; latency, less bandwidth and higher power consumption. In order to overcome the electrical bottleneck, all optical networks (AON) have been proposed. In AON, optical processors are used, which process the data in the optical domain. This requires improvement in optical control plane protocols. Therefore, GMPLS protocol has been proposed, [1] which improves the optical network utilization and simplifies the AON implementation. With the use of a GMPLS control plane at the physical level, the asynchronous transfer mode and Internet protocol layers are merged, as shown in Fig.1, to provide more transparency and dynamic re-configurability. It supports packet switching along with time, space and wavelength switching. The key element of transparent optical network is the GMPLS router. We have proposed the micro-resonator based GMPLS router for optical network on chip $(\mathrm{ONoC})$, with which the existing system on chip (SoC) will be able to give maximum throughput with minimum blocking probability. The micro-resonators can be active or passive in nature. The active micro-resonator is one whose resonance wavelength is controlled by voltage or current source. The passive micro-resonators are those whose resonance wavelength is a property of the material being used for it and its geometrical dimensions. We have studied passive type micro-resonator. Various structures of ONoC are proposed [2-4], which deal with the physical as well as system level design issues.

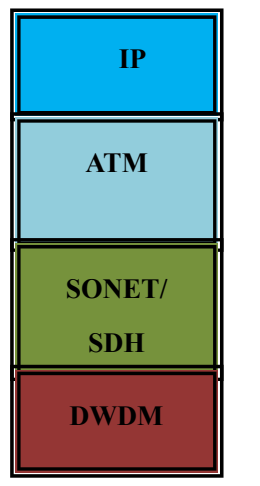

Traditional Optical Network Stack

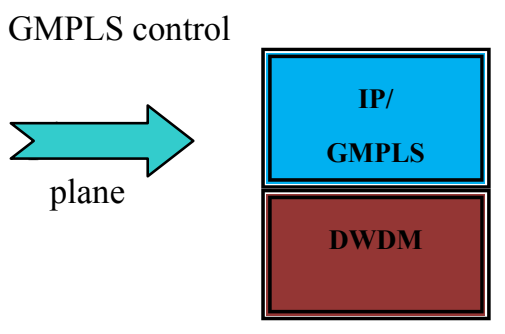

GMPLS Optical Network Stack

Figure1. Multilayer data network evolved as a GMPLS core network. 
The study showed a future scope in physical level analysis based on physical impairments such as crosstalk and other fiber losses. For the first time we have proposed the micro resonator based GMPLS router, crosstalk and blocking probability analysis for ONoC at the physical level. In [1] authors have proposed a novel labeling scheme for GMPLS network and their results reported, indicate that decreasing the upper bound of the connected paths will reduce the probability of the performance degradation. Our results show that by reducing the Bit error rate (BER) of an ONoC higher accessibility is achieved. A mesh based $\mathrm{ONoC}$ is studied to find out the minimum signal to noise (SNR) links [2]. In this paper we studied the relationship between the network size in terms of number of OXC stages, crosstalk, optical SNR, and BER and found the possible number of routing stages in an ONoC with acceptable OSNR. The rest of the paper is organized as follows. In Section 2 the architecture of micro-resonator based GMPLS router for ONoC is described. In Section 3 traffic behavior of the $\mathrm{ONoC}$ is studied. Section 4 discusses the results reported and Section 5 concludes the paper.

\section{Optical Network on Chip architecture}

Architecture of an optical network on chip (ONoC) is shown in Fig.2, which consists of mainly three types of blocks. (1) Encoder - Multiplexer: it is the electrical to optical (E - O) Interface. This block is generally composed of directly modulated laser sources, along with the associated driver circuits and multiplexers. Input to the encoder is from a generic NoC protocol converter (GNoCPC). There exists a serial data channel between the converter and the laser source. (2) $\lambda$-Router: the key element in ONoC domain. The structure of $\lambda$-router is shown in Fig. 3(a), which consists of second order $2 \times 2$ micro-resonator, Fig.3 (b) [2, 4], for routing the data between SoC components.

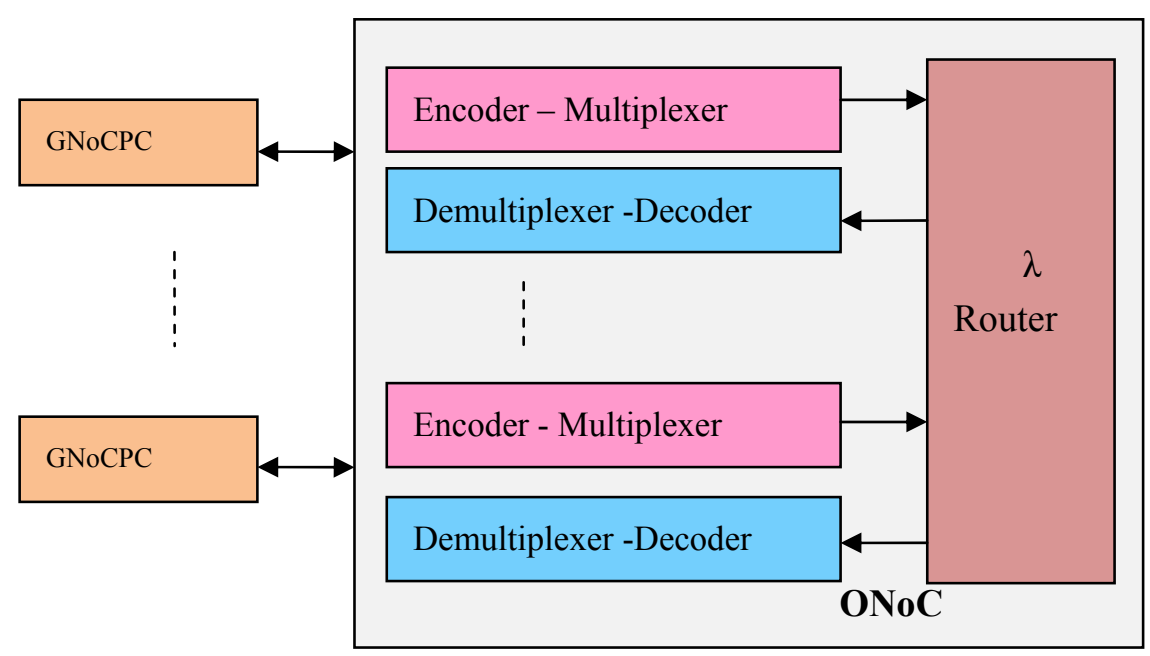

Figure2. Architecture of Micro-resonator based GMPLS router ONoC

It has initiators (I) and targets (T). (3) Demultiplexer - Decoder: this acts as optical to 
electrical $(\mathrm{O}-\mathrm{E})$ Interface. It comprises of decoder, photo-detector and trans-impedance amplifier. The structure of $\mathrm{ONoC}$ is heterogeneous, which is composed of active and passive optical components and analog / digital integrated circuits. An $\mathrm{N} \times \mathrm{N} \lambda$-router structure is used, where $\mathrm{N}$ is the number of input or output ports. We have considered $\mathrm{N}=8$, then the router will have 8 initiators (I) and 8 targets (T) shown in Fig. 3 (a). The basic structure of second order micro-resonator is shown in Fig. 3 (b). There are three different switching states depending on the input signal, they are as follows: (1) Bar state: Occurs when the resonant wavelengths are injected in the filter and routed into the through port. (2) Cross state: When the non-resonant wavelengths are injected in the filter and are dropped out. (3) Cumulative state: When both resonant and non-resonant wavelengths are injected into the filter using WDM technique and are either routed or dropped by the resonator. The wavelengths can be injected from any input initiator and the switching is obtained at any one of the output targets, depending upon the wavelength value of the optical signal being injected.
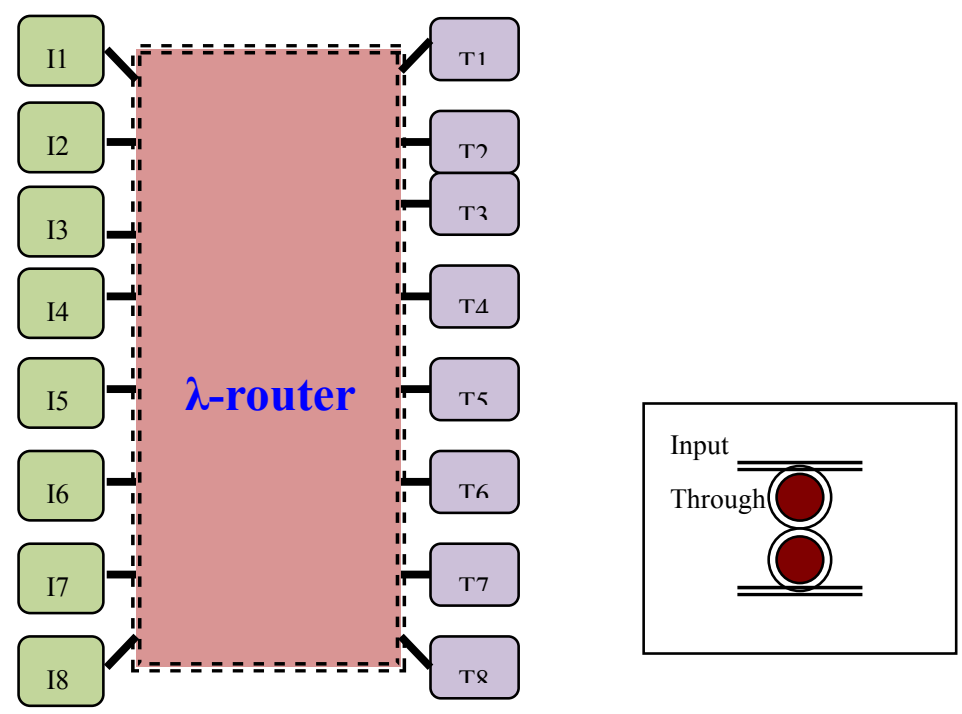

Figure3. (a) $8 \times 8 \lambda$-router architecture. (b) 4 port micro resonator

The input initiator can communicate with the target by sending the encoded data packet routed through the GMPLS micro resonator based router. While routing through the router, there exists crosstalk between the various wavelength division multiplexing (WDM) channels due to its imperfect resonance characteristics. Crosstalk is an intrinsic characteristic of micro resonators and waveguide crossings. There can be two types of crosstalk, depending on the wavelength of the source; Inter band (incoherent) or Intra band (coherent) [5]. The amount of crosstalk noise accumulated at each router goes on increasing, which results in more error probability in the received packets. This in turn increases the blocking probability and packet error rate. We have reported the dependency of system blocking probability on the crosstalk and packet length. 


\section{Analytical Modeling}

In GMPLS technology with total $M$ channels at the input, each of WDM channel is provided with a specific label $C_{i}$ enters at $I_{i} i^{\text {th }}$ initiator. The connection request is placed from the initiator to the respective target, with an assumption that, the connection request arrival to be a random process, modeled as Poisson distribution using well-known Erlangs traffic Model. Considering its mean ' $m$ ' and holding time of the connected path with mean ' $\mu$ ' then the probability of getting path connection is given as [6]

$$
P_{X}(x)=\frac{P_{0}}{x !}\left(\frac{m}{\mu}\right)^{x} \text { where } x=0,1, \ldots M
$$

Where, $P_{X}(x)$ is the PDF of ' $x$ ' and $P_{0}$ is obtained considering the normalized condition $\sum P_{x}(x)=1$ as

$$
P_{0}=\frac{1}{\sum_{k=0}^{m} \frac{l^{k}}{k !}} \text { where } l=m / \mu \text { offered traffic load }
$$

At the receiver target, a signal is detected using decoder and photo detector combination. Assuming a Gaussian noise approximation the detector error probability $\left(P_{e}\right)$ is found, which further helps in defining the blocking probability, $P_{b}$ in the $\mathrm{ONoC}$ as in (3).

$$
P_{b}=\sum_{k=1}^{M} P_{k}(k) P_{e}(k)
$$

Assuming the average packet length $L_{i}$ bits the packet error rate (PER) can be obtained as

$$
P E R=1-\left(1-P_{b}(k)\right)^{L_{i_{i}}}
$$

\section{Results and Discussions}

Fig. 4 shows the plots for number of GMPLS router stages versus OSNR. We find that as the network size increases, the optical signal power received by the destination system drops quickly, and the crosstalk power is relatively high, due to the accumulation of crosstalk at successive stages. This results in limited network size. Conversely, if the crosstalk is limited 


\section{Macrothink}

to $-30 \mathrm{~dB}$ then the better performance with respect to OSNR and network size is observed. Minimum of $2 \mathrm{~dB}$ OSNR is achievable when the optical signal passes through 20 stages of routers for the crosstalk level of $-20 \mathrm{~dB}$. Fig. 5 shows the impact of crosstalk on network blocking probability, as a function of packet length. We define here a term called flit length also known as packet length which plays an important role in ONoC design. A moderate packet length of 300 bits shows good performance at higher crosstalk levels. Increasing packet length at higher crosstalk values, results in higher blocking probability. Our results show that with 8 wavelengths in WDM channel, $1 \%$ blocking exists when the crosstalk level is $-10 \mathrm{~dB}$. Fig. 6 shows the plots of the blocking probability as a function of traffic load for the various crosstalk levels.

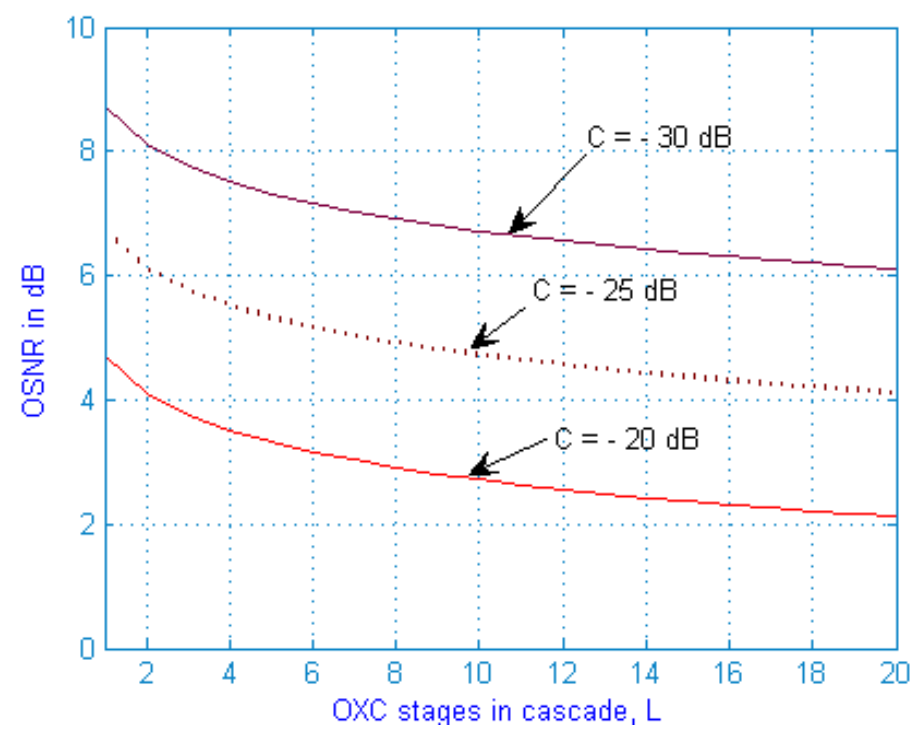

Figure4. Router stages versus OSNR

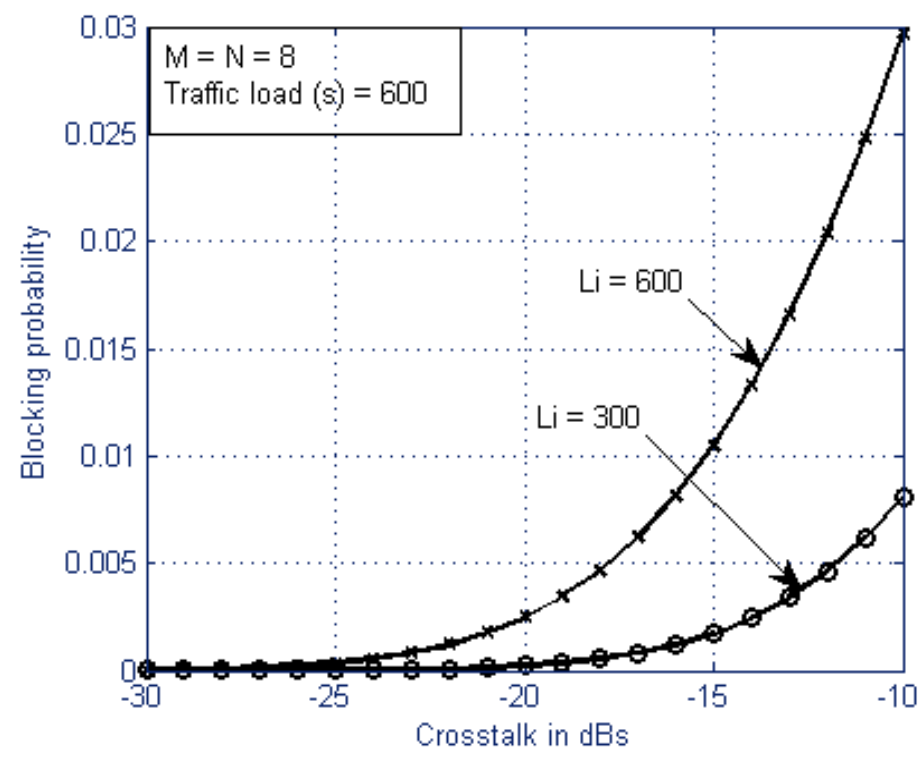

Figure5. Crosstalk versus blocking probability 
The PER against the crosstalk levels for different packet length is shown in Fig.7. Here the number of wavelengths are 8, carrying traffic of 600 Erlangs. As the crosstalk increases, the noise associated with the detector also contributes to the PER.

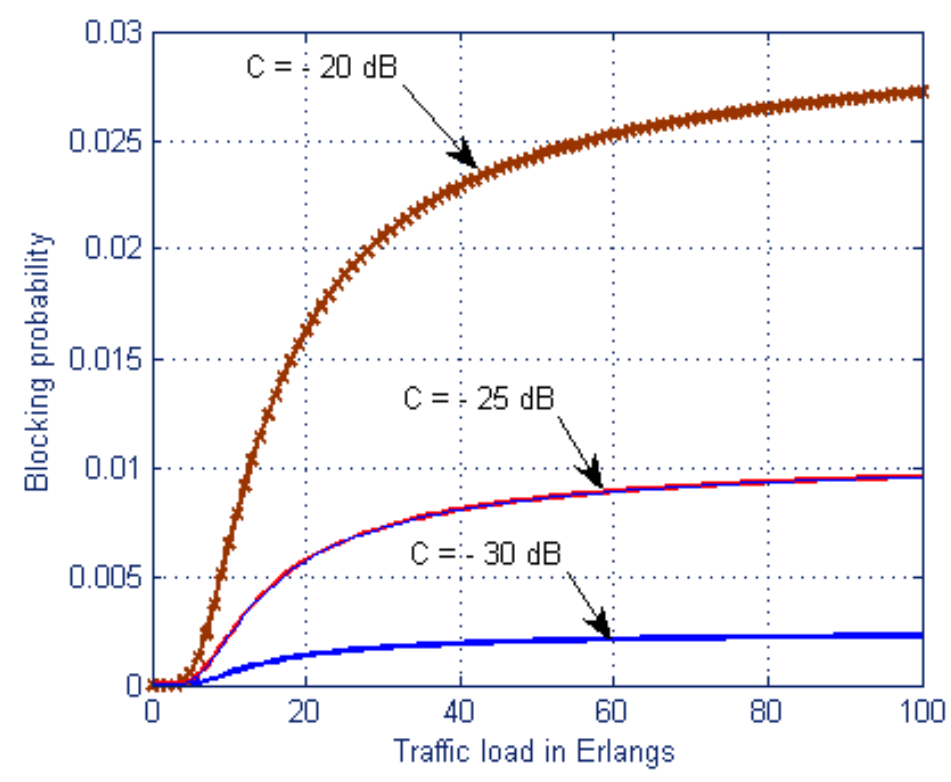

Figure6. Offered traffic load versus blocking probability

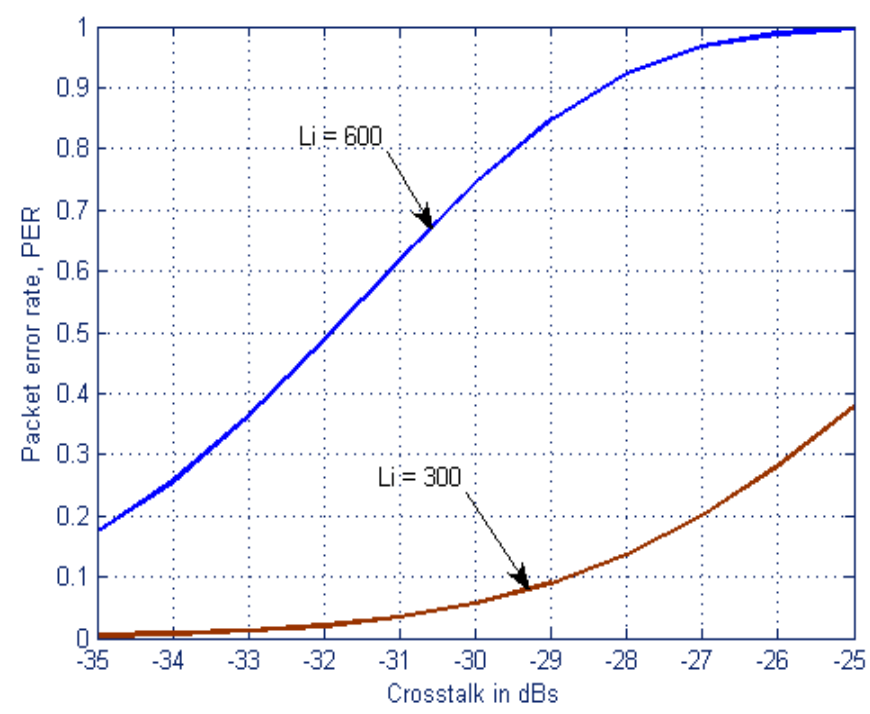

Figure7. Plots of PER of incoming traffic against crosstalk

\section{Conclusion}

A novel micro resonator based GMPLS router structure for ONoC design is investigated on the basis of crosstalk, blocking probability and packet error rate. Crosstalk being the intrinsic characteristics of a wavelength router is small, at the device level but when 
considered at the system level, showed significant impact. Our results show that crosstalk becomes a major limiting factor for the transmitted packet length and non-blocking condition in the GMPLS ONoC. Heavy traffic load of 600 Erlangs with a packet length of 300 bits can be used at $40 \%$ PER. The crosstalk level of $-30 \mathrm{~dB}$ is tolerated for higher traffic load condition to obtain minimum blocking probability. Crosstalk analysis for $\mathrm{ONoC}$ would help in designing the GMPLS networks with good QoS for providing multi service facility, and also in deciding the number of routing stages in the networks with acceptable performance. Minimum of $2 \mathrm{~dB}$ OSNR is achievable when the optical signal passes through 20 stages of routers for the crosstalk level of $-20 \mathrm{~dB}$.

\section{References}

[1] Hamzeh Beyranvand, and Jawad Salehi., "Multiservices Provisioning and Quality of Service Guarantee in WDM Optical Code Switched GMPLS Core Networks". Journal Of Lightwave Technology. Vol. 27, Issue 12. Pp. 1754 - 1762. June 2009. http://ieeexplore.ieee.org/10.1109/JLT.2009.2017499.

[2] Yiyuan Xie, Mahdi Nikdast, Jiang Xu, Wei Zhang, Qi Li, Xiaowen Wu, Yaoyao Ye, Xuan Wang, Weichen Liu., "Crosstalk Noise and Bit Error Rate Analysis for Optical Network-on-Chip”. Design Automation Conference (DAC'10). 2010. http://ieeexplore.ieee.org/iel5/5510861/5522347/05523371.

[3] O’Connor, F. Tissafi-Drissi, D. Navarro, F. Gaffiot, J. Dambre, M. Wilde, D. Stroobandt, M. Briere., "Integrated Optical Interconnect for on-chip Data Transport". IEEE, North-East Workshop on Circuits and Systems. Pp. 209-212. 2006.

http:// ieeexplore.ieee.org/10.1109/22.508632.

[4] Lei Zhang, Mei Yang, Yingtao Jiang, Emma Regentova, Enyue Lu. "Generalized Wavelength Routed Optical Micronetworks in Network-on-chip". $10^{\text {th }}$ IASTED International Conference on Parallel and distributed Computing and systems. Pp. 698-703. Nov. 13-15, 2006. http:// ieeexplore.ieee.org/+DOI.

[5] Hercules Simos, Charis Mesaritakis, Dimitris Alexandropoulos, and Dimitris Syvridis, "Dynamic Analysis of Crosstalk Performance in Microring-Based Add/Drop Filters", Journal of Lightwave Technology, Vol. 27, No. 12, Pp. 2027-2034. June 15, 2009. http://ieeexplore.ieee.org/10.1109/JLT.2008.2006508.

[6] S.S. Patil, B. S. Chaudhari and Baojun Li, "Analysis of Crosstalk and Modeling of Possible Number of Routing Stages for Optically Transparent WDM GMPLS Networks". Journal of Optical Engineering. SPIE. Vol.no.49. Pp. 115001-115006. 2010. http://spiedl.org/10.1117/1.3509193.

\section{Copyright Disclaimer}

Copyright reserved by the author(s).

This article is an open-access article distributed under the terms and conditions of the Creative Commons Attribution license (http://creativecommons.org/licenses/by/3.0/). 